\title{
Einiges über Samuel Thomas Sömmering und seine Zeitgenossen
}

Von J. Karcher, Basel

Mitten im politisch verworrenen, von gegensätzlichen geistigen Strömungen wechselseitig bewegten Zeitabschnitt des zu Ende gehenden 18. Jahrhunderts bildete Kassel ein Kulturzentrum besonderer Art. Der regierende Markgraf Friedrich II. war ein leidenschaftlicher Sammler von Altertümern und Raritäten; er liebte den Verkehr mit Gelehrten und Künstlern und förderte sie, wo er konnte. Er gründete eine Malakademie und ließ seinen Minister von Schlieffer gewähren, als er eine auserlesene Schar jugendlicher Wissenschafter an die alte Carlsschule von Kassel berief. 1779 erhielt Georg Forster, einer der genialsten Vertreter der Aufklärung in Deutschland, der sich durch die Berichte über seine Forschungsreise mit dem Weltumsegler Соoк einen wissenschaftlichen und schriftstellerischen Ruf erworben hatte, den Lehrstuhl für Naturwissenschaften und Samuel Thomas Sömmering die anatomische Professur als Nachfolger des 1778 verstorbenen Johann Jаков Huber, jenes gebürtigen Baslers, der Prosektor bei HaLler in Göttingen gewesen war. 1781 erweiterte sich der Kreis der jungen Gelehrten durch die Berufung von Johannes Müller als Historiker.

Sie mußten alle mit einem niedrigen Gehalte vorliebnehmen; sie darbten, um standesgemäß ihren höfischen Verpflichtungen nachkommen zu können. Der Fürst gab ihrer angusta res domus beachtenswerte Kompensationen. Er verschaffte Sömmering eine Amtswohnung und ein wohlausgestattetes anatomisches Theater. Die markgräfliche Menagerie und das ihr angegliederte Negerdorf lieferten Sömuering ein unvergleichliches Forschungsmaterial. Er war in Göttingen bei WrISBERg und dessen Schüler Blumenbach und Loder im Anatomisieren geschult worden; dann hatte er sich bei Peter Camper und John Hunter im Sezieren weiter ausgebildet. In Kassel erwies er sich sehr bald als ausgezeichneter Zergliederer und wurde als solcher von den hohen Gästen des Fürsten, vor allem vom Herzog von Weimar und dem ihn begleitenden GoEthe, anerkannt.

Goethe empfand es dankbar, daß Sömmering seinen Knochenstudien Verständnis entgegenbrachte und ihn anhand seiner Sammlungsobjekte an seinen anatomischen Forschungen teilnehmen ließ. Über das freundschaftliche Verhältnis der beiden zueinander legt ein bis zum Jahre 1828 fort- 
geführter Briefwechsel ein beredtes Zeugnis ab. Es sind sachliche, von gegenseitiger Achtung getragene Briefe, in denen Gozthe seinen Freund um die wunderlichsten Schädelsendungen angeht. Sömmering sandte Elefanten-, Rhinozeros-, Kamel- und andere Schädel nach Weimar und Eisenach oder zu Frau Rat Goethe nach Frankfurt. Einmal kam das schöne Verhältnis in Gefahr, getrübt zu werden: GoEтнe hatte sich von Sömmering einen Kamelsschädel erbeten. Als nach einiger Zeit Sömmering den säumigen Freund an seine Rückgabe mahnte, erhielt er den Schädel beschädigt mit einem falschen Unterkinnbacken zurück; der richtige Unterkiefer blieb unauffindbar.

Unter den Schaustücken der Sammlung Sömmerings befand sich eine Anzahl von Mißgeburten, die der Markgraf für teures Geld gekauft und ihm gestiftet hatte. Goethe widmete ihnen bei seinem Besuch besondere Aufmerksamkeit. Söмmering beschrieb sie in einer 1791 in Mainz erschienenen Publikation, in der er sich bemühte, «eine Stufenfolge und natürliche Ordnung selbst in den Mißbildungen unseres Körpers zu entdecken und darzutun». Die darin vertretene Betrachtung der Mißbildungen als Metamorphosen bildete offenbar schon 1783 den Gesprächsstoff zwischen GoEthe und Sömmering. Unser leider so früh in den Bergen tödlich verunglückter Kollege Sebastian Hodel hat 1948 seine Inauguraldissertation über diesen Gegenstand geschrieben; ich sehe darum davon ab, hier darauf einzugehen. - Aus den Briefen Goethes an Sömmering geht hervor, daß er mit dem Ergebnis seines Besuches in Kassel zufrieden war. Dieser Besuch war übrigens ein Teilstück seiner Rundfahrt zu den Anatomen Blumenbach, Camper, Merck und Loder, die er zur Dokumentierung seiner Zwischenkieferarbeit zu benötigen glaubte. Bekanntlich kam er schließlich mit dem Jenenser Anatomen LoDER, zu dessen regelmäßigen Hörern er gehörte, zum Ziel (1784).

Sömmering war auf die Befürwortung Forsters nach Kassel berufen worden. Die beiden Freunde Georg Forster und Thomas Sömmering bildeten ein Zweigespann von eigenartigem Format. Söмmering war mit Forster zusammengekommen, als er studienhalber in London weilte. Seitdem waren sie unzertrennliche Freunde geworden.

Sie waren gleichen Alters, beide 1754 auf polnischem Boden geboren: Samuel Thomas Sömmering als Sohn des Stadtarztes von Thorn, Georg Forster als der Sohn des Pastors Reinhold Forster im Dorfe Nassenhuben bei Danzig. Er stammte in vierter Generation von englischen Flüchtlingen aus der Zeit Cromwelus ab. - Sie waren beide in London Freimaurer 
geworden. Sie hofften, bei diesen die Ideale verwirklicht zu finden, die in deren Satzungen niedergelegt waren. Es waren dieselben, die Lessing in seiner Schrift Ernst und Falk (1778) wiedergibt und die er seinem Nathan dem Weisen (1780) zugrunde legt. Forster und Sömmering wurden durch die menschlichen Unzulänglichkeiten der Logenbrüder in Kassel enttäuscht. So kam es, daß sie den Rosenkreuzern in die Arme liefen, bis sie sich auch von diesen lossagten, von ihrer unsauberen alchemistischen Goldmacherei angewidert und ausgeplündert. Die Nachwehen dieses Rosenkreuzerhandels trübten ihr Leben während einiger Jahre.

Was der Geheimbund der Rosenkreuzer im 18. Jahrhundert wirklich gewesen ist, darüber weiß niemand etwas Unzweideutiges zu berichten. Keine Quelle vermittelt eine verläßliche Auskunft. Die Rosenkreuzer waren ursprünglich ein frommer theosophischer Orden gewesen. Zur Zeit der Aufklärung, da Philosophie, Skepsis oder bloße Frivolität den biblischen Offenbarungsglauben und die sittliche Lebensführung unterwühlten, erschien er manchen als ein Zufluchtsort für ihre Einstellung zur Transzendenz, für ihren Drang zur Weltverbesserung. So läßt es sich vielleicht erklären, daß sich Georg Forster, Sömmering und Johannes MüLler den Rosenkreuzern zuwandten. Am traditionellen Glauben irre geworden, zugleich aber weltanschaulich noch unreif, waren sie ihren Lockungen anheimgefallen.

Sie hatten das Glück, daß ihnen Gottlob Heyne (1729-1816), der Redaktor des «Göttinger gelehrten Anzeigers», die Verbindung mit der Universität Göttingen vermittelte, indem er sie mit der Rezension von Büchern beauftragte. Heyne war der freundschaftlich beratende Genius des geistessprühenden Kasseler Trios. Als Forster seine Tochter Therese heiratete, wurde das Verhältnis noch vertraulicher. Sömmering blieb Heyne bis zu dessen Tode treu zugetan.

Die drei Kasseler Freunde wurden bald auseinandergerissen. Forster folgte 1784 einem Rufe an die russisch-polnische Universität Wilna. Während der Zeit ihrer Trennung haben Forster und Sömmering regelmäßig miteinander korrespondiert. Dieser Briefwechsel ist später veröffentlicht worden. Er hat bis heute seine Lebensfrische behalten. Kein Leser kann sich dem gewinnenden Wesen und der edlen Gesinnung Forsters entziehen. Man begreift, daß Goethe Georg Forster als etwas Besonderes einschätzte.

Johannes Müller war als erster nach Mainz übergesiedelt. Er hatte das Amt eines Bibliothekars der kurfürstlichen Bibliothek übernommen. 
Sömmering war ihm schon 1784 gefolgt. Als dann Müller Staatsrat wurde, bemühte er sich um die Berufung Forsters an seine Stelle. Forster kam 1788 nach Mainz.

Die ersten Jahre ihres Mainzer Zusammenseins waren der intensivsten Arbeit bei bestem Einvernehmen gewidmet. FonSTER lieferte wertvolle schriftstellerische Arbeiten. Sömmering bemühte sich um die Herstellung anatomischer Präparate und ihre Darstellung in Modellen und Zeichnungen. Er hatte in Christian Koeck einen Künstler gefunden, der ihm dabei behilflich war. Forsters Haus war der Treffpunkt der drei Freunde.

Erst als die Anzeichen eines drohenden revolutionären Umbruches sich auch in Mainz mehrten, machten sich gegensätzliche Einstellungen bei ihnen geltend. Beseelt vom Optimismus seiner rationalistischen Weltanschauung, fühlte sich Forster zu den von der Französischen Revolution proklamierten idealen Zielen hingezogen. Ihm schwebte eine ähnliche Befreiung für das deutsche Volk vor. Bei Sömmering trat dagegen sein ausgesprochen deutsches Wesen, gepaart mit einer unüberwindlichen Antipathie gegen das Franzosentum, in Erscheinung und wurde übermächtig, als er sich in Frankfurt mit Elisabeth Grunelius, der Tochter einer angesehenen Bankierfamilie, vermählte.

Als sich Goethe 1792 auf der Durchreise zur alliierten Armee in Mainz auf hielt, verbrachte er mit «Sömmerings, Forsters und Huber» (LudwiG Ferdinand Huber, der ästhetische Kritiker und Sekretär des kursächsischen Gesandten in Mainz) «zwei muntere Abende». Man unterhielt sich über Kunst, Philosophie, Wissenschaft, vermied politische Dinge, «da man fühlte, daß man sich wechselseitig zu schonen habe».

Ein Bruch zwischen Sömmering und Forster trat ein, als Sömmering von Frankfurt aus zur Zeit der französischen Besetzung von Mainz von Forster unmöglich zu erfüllende Schutzmaßnahmen für seine Sammlungen forderte und damit den verzweifelt kämpfenden Freund empfindlich verletzte.

Der Einbruch der Französischen Revolution in deutsches Gebiet hatte aus Mainz einen Hexenkessel politischer Gegensätze und kriegerischer Zerstörungen gemacht. Forster war dabei ein Opfer seiner Freiheitsideale, seiner überpolitischen Ethik, seiner Einsatzbereitschaft für die sich ihm aufdrängenden sozialen und humanitären Verpflichtungen geworden. Die Mainzer vergalten ihm all das, was er während der französischen Besetzung mit dem Einsatz seiner selbst für sie getan hatte, mit Undank, $\mathrm{Ha} ß$ und Verfolgung. Er starb eines jammervollen Todes, von allen verlassen, in 
einem armseligen Hotelzimmer in Paris, enttäuscht von der menschlichen Unzulänglichkeit und der Ruchlosigkeit der großen und kleinen Akteure der Schreckensherrschaft. Therese Forster war aus Mainz in die Schweiz geflüchtet.

Sömmering hat Forster nachgetrauert. Zusammen mit Forsters Schwiegervater Heyne nahm er sich seiner Frau und seiner Kinder an, versuchte aus seinem Nachlasse zu retten, was zu retten war, und übernahm die Vormundschaft über Forsters Kinder.

Georg Forster hinterließ zwei Töchter, von denen die eine den Berner Gottlieb von Greyerz heiratete. Von diesem Paare Greyerz-Forster stammt die Familie von Greyerz ab, die somit Georg Forster zum Ahnherrn hat.

Als sich Goethe am Tage, da der Waffenstillstand zwischen der Mainz belagernden alliierten Armee und der französischen Garnison abgeschlossen wurde, der Stadt näherte, stieß er unversehens auf Sömmering. Dieser war von Frankfurt herbeigeeilt, um nach seinen Sammlungen zu sehen. Als andern Tags die beiden das Haus Sömmerings betraten, fanden sie wohl die Zimmer, in denen sie vor einem Jahre muntere Stunden verlebt hatten, übel zugerichtet; aber im Keller waren die Sammlungen Sömmerings unbeschädigt.

Diese Sammlungen lagen Sömmering am Herzen, weil sie die Präparate enthielten, nach denen er seine großen Tafelwerke über die Sinnesorgane (Auge 1801, Ohr 1806, Geschmack und Stimme 1806, Geruch 1809) verfassen und illustrieren wollte. Er ließ sich in diesen Arbeiten von den gleichen strengen Grundsätzen der Morphologie leiten, die er bei der Abfassung seines Lehrbuches vom Bau des menschlichen Körpers (1791) befolgt hatte. Das schönste Werk dieser Art ist seine weibliche Skelettfigur (1797), die in ihrer Vollkommenheit der Auffassung und Darstellung an Vesalsches anatomisches Arbeiten erinnert. Sömmering vertrat den Standpunkt, daß jede abgeschlossene wissenschaftliche Arbeit, die man dem Publikum vorlegt, ein Kunstwerk sein müsse.

Die mörderischen Zeitläufte lieferten SöмMERING ein ausgiebiges Leichenmaterial, das ihm erlaubte, seine Sektionstechnik zu verbessern. Es gelang ihm, im Gehirne die «Nervenenden» (Nervenwurzeln) weitgehend zu verfolgen, die Autonomie des Sympathicus darzulegen, am senkrechten Durchschnitt, d. i. «am wahren sogenannten Profile des Hirnes», die Hirnhöhlen naturgetreu darzustellen. Er konnte, im Gegensatz zu Haller, zeigen, daß die Flüssigkeit der Hirnhöhlen zum natürlichen Bau des Gehirnes ge- 
höre. Alle diese Befunde legte er in seiner 1796 erschienenen Schrift Über das Organ der Seele nieder. Sömmering widmete sie «unserm Kant».

Immanuel Kant hatte 1781 mit seiner Kritik der reinen Vernunft den Deutschen eine spezifisch deutsche, der ureigenen Struktur ihres Geistes adäquate Philosophie geschenkt. Er lehrte sie philosophisch denken, indem er ihnen dafür Regeln bot, die er, befreit von metaphysischen Deutungen, den Prinzipien der Vernunft entnahm. Theoretischer und praktischer Idealismus lag seinem Streben nach Erkenntnis zugrunde. Die Philosophie Kants ist allerdings eine tiefe und schwierige Lehre. Sie ist im Laufe der Zeit auf mancherlei Weise gefaßt und dargestellt worden. KANT ist auf den glücklichen Einfall gekommen, seiner Kritik der reinen Vernunft Prolegomena anzufügen; er dachte sie denjenigen $\mathrm{zu}$, die in ihrem Geiste ein Quäntchen Brennstoff haben, den ein Funke KavTscher Verkündigung zu entzünden vermag. Diese Prolegomena und die Biographie, die Friedrich Wilhelm Schubert den Sämtlichen Werken Kants 1842 beigegeben hat, bilden die Hauptquelle zu meinen Ausführungen über Kant. Ich greife aus der Fülle der Kantschen Gedankenwelt einen einzelnen Punkt heraus, nämlich seine Erkenntnis a priori und seine Einladung an die Naturforscher und Ärzte, «statt ihre Erkenntnis nach den Gegenständen sich richten zu lassen, einmal zu versuchen, ob man nicht besser fortkomme, wenn man annehme, die Gegenstände müssen sich nach unserer Erkenntnis richten». In seinen Prolegomena (§18) beantwortet Kant die Frage: «Wie ist reine Naturwissenschaft möglich ?» dahin, daß er sagt: «Empirische Urteile, sofern sie objektive Gültigkeit haben (er nennt sie Erfahrungsurteile), erfordern jederzeit über die Vorstellung der sinnlichen Anschauung hinaus noch besondere im Verstande ursprünglich erzeugte Begriffe, welche es eben machen, daß das Erfahrungsurteil objektiv gültig sei.» Dabei ließ Kant der empirischen Realität ihr volles Recht und erkannte der Mathematik im wissenschaftlichen Aufbau der Naturlehre ihre volle Bedeutung zu. In $\S 35$ der Prolegomena mahnt er, die Einbildungskraft in Schranken zu halten: «Wenn der Verstand, der denken soll, statt dessen schwärmt, so kann ihm das niemals verziehen werden ....

Er konnte es aber nicht verhüten, daß seine auf die Erkenntnis a priori gerichtete Lehre auf solche Mediziner, die mit Vorliebe der aprioristischen Meditation zugeneigt waren, verführerisch wirkte, so daß sie sich seiner Mahnung zum Trotz zu eben diesem Schwärmen verleiten ließen. Es entstand ein eigenartiges medizinisches Schrifttum, für das das Buch Söмmerings Über das Organ der Seele ein prägnantes Beispiel darstellt. 
Sömmering stellte in dieser Schrift a priori den Satz auf, daß der «gemeinschaftliche Empfindungsort» (das sensorium commune) sich in der Feuchtigkeit der Hirnhöhlen befinde. Als Beweis dafür konnte er aufzeigen, «daß die Hirnnervenenden oder die wahren Ursprünge der meisten, wo nicht aller Hirnnerven sich an den bestimmten beständigen Stellen, auf den Wänden der Hirnhöhlen, zeigen oder sich bis auf die Wände der Hirnhöhlen so verfolgen lassen, daß man sagen darf: die Hirnenden der Nerven oder die wahren Ursprünge der Nerven werden von der Feuchtigkeit der Hirnhöhlen an bestimmten Stellen berührt, und wechselseitig - oder umgekehrt: die Ursprünge der Nerven berühren an bestimmten Stellen die Feuchtigkeit der Hirnhöhlen; - oder zwischen den Nervenenden und der Feuchtigkeit der Hirnhöhlen findet Wechselberührung statt».

Mit dem sensorium commune hat es nun folgende Bewandtnis. Seitdem Descartes im 17. Jahrhundert die Zirbeldrüse als die Kontaktstelle der immateriellen Seele mit dem materiellen Leibe bezeichnete, mühten sich die Anatomen und Physiologen damit ab, im Gehirne das wirkliche Organ der Seele aufzufinden. Unter dem Einflusse der sensualistischen Philosophie von John Locke setzten sie an die Stelle der unsterblichen Seele das sensorium commune, d. i. die «gemeinschaftliche Empfindungsstelle» (SöмMERING) oder der "gemeinsame Empfindungsplatz» (Kant) in Ansehung der Sinnesempfänglichkeit und des Bewegungsvermögens der Seele.

Sömmering fand im anatomischen Schrifttum zahlreiche Lösungen dieser Frage, von denen keine ihn befriedigte. Hier seien nur einige der wichtigsten unter ihnen genannt: LANGISI und Bonnet verlegten das sensorium commune in den Balken, Vieussens in das centrum semiovale, Willis in das corpus striatum, Drelincourt in das Kleinhirn, Albrecht von Haller in den pons, andere wieder in die Hirnscheidewand oder in die Vierhügel oder gar in das Rückenmark (Mieg). Sömmering fand es unbegreiflich, daß man das sensorium commune in einem soliden, starren oder rigiden Teile des Hirns suchen konnte. Es widerstrebte ihm anzunehmen, daß es auf einen kleinen Teil des Hirnmarkes eingeschränkt sein sollte. Nach seiner Meinung mußte es einem vereinigenden Mitteldinge (medium uniens) innewohnen, mit dem alle Nervenendigungen in Berührung kommen. Das konnte nur die Flüssigkeit der Hirnhöhlen sein.

Nun ergab sich aber für Sömmering folgendes unabweisliches Postulat: die Hirnflüssigkeit konnte ihre Aufgabe als «gemeinschaftliches Sensorium» nur dann erfüllen, wenn sie animiert, d. i. belebt war. Das zu beweisen, führte ihn in die fernsten Gefilde der Metaphysik, der Transzendenz. Er 
argumentierte mit dem Geiste Gottes, der in der Genesis auf den Wassern schwebte, rief Aristoteles und Thales, der das Göttliche im Wasser zu finden glaubte, zu Hilfe. Er fand bei den «tiefsten, erfahrensten, echtesten Denkern» Lfibnitz, Newton, Euler, Kant seine Ansicht bestätigt, daß das Animiertsein - Belebtsein - einer Flüssigkeit nicht nur wahrscheinlich, sondern zu den Erscheinungen des Lebens selbst notwendig sei.

Dann zog er zur Stütze seiner Lehre vom Hirnwasser als dem Organ der Seele Schlußfolgerungen aus der Analogie (Augenwasser, Labyrinthwasser usw.) heran, baute überlegungsmäßig kunstvolle Konstruktionen auf, die er der Embryologie, der Physiologie, der Pathologie oder der vergleichenden Anatomie entnahm, und suchte bei den Autoren Stellen, die seiner Theorie günstig zu sein schienen. Sie haben weder Zeitgenossen noch Nachfahren zu überzeugen vermocht. Ich verzichte darauf, sie hier wiederzugeben.

Sömmering war nach den schicksalsschweren Ereignissen von 1792/93 vereinsamt zurückgeblieben. Forster war tot. Johannes MüLler befand sich in Wien. Voller Bitterkeit formulierte er seine Widmung affektbetont «unserm Kant».

Er war mit der Philosophie KANTs bekannt geworden, als begeisterte Verkünder derselben sie an den süddeutschen Universitäten zu lehren begonnen hatten. In Mainz hielt Professor Dorsch 1789 Vorlesungen über das philosophische System Kants. Mit seinen Werken gelangte auch die Kunde von seinen überragenden menschlichen Charaktereigenschaften nach Süddeutschland. JAсовI, der Philosoph von Pempelfort, schrieb 1788 an Forster: «Kant verehre ich als einen Mann von außerordentlichem Geiste.» Georg Fonster war allerdings Kant nicht restlos zugetan. Er kritisierte seinen schwerfälligen Stil und erhob sich gegen seine Auffassung von den Menschenrassen. Er und Johannes Müller fühlten sich mehr zu zu den Ideen Herders hingezogen.

Als Goethe 1792 in Mainz weilte, lag die kritische Philosophie Kants noch außerhalb seines Blickfeldes. Fr gibt zu, sich bis 1794 wenig mit ihr abgegeben zu haben. Erst seit seinem vertrauteren Umgang mit Schiller (1794) gewann er umfassende Kenntnisse von ihr, anfangs gesprächsweise, später durch die Lektüre der Kantschen Kritik der Urteilskraft. ScHiller regte auch Wilhelm von Humboldt an, Kant zu studieren. Die Begeisterung Humboldts für Kant hat viel dazu beigetragen, seinen Ruhm in der Welt der Gelehrten zu festigen.

Um so unverständlicher erscheint es heute, daß um die gleiche Zeit, im Gegensatze zu dieser allgemeinen Anerkennung, ausgerechnet von Berlin 
aus obskurantistisch eingestellte Zensoren unter Friedrich Wilhelm II. dem Königsberger Philosophen unwürdige Plackereien und Demütigungen zuteil werden ließen, die ihm, dem Siebzigjährigen, seelisch und körperlich zusetzten. In diese für ihn trübe Zeit fiel die Widmung von Sömmerings Buch über das Organ der Seele. Sie scheint auf Kant einen erfrischenden Eindruck gemacht zu haben. Er schrieb umgehend an den Verfasser einen anerkennenden Brief, in dem er ihn seiner Wertschätzung und freundschaftlichen Verbundenheit versicherte.

Kant meinte, daß das Responsum auf die Frage nach dem Sitz der Seele durch die Verschiedenheit der Prämissen im Denken der Mediziner und der Philosophen bedingt sei. Die Mediziner gründeten ihre Schlüsse auf empirische Prinzipien, die Philosophen als Metaphysiker zuoberst auf Gründe a priori. Trotzdem bemühte er sich, den Gedankengängen Sömmerings weitgehend zu folgen, und schlug ihm zur Stütze der Annahme, daß das Hirnwasser der Sitz des sensorium commune sei, eine abenteuerliche Hypothese vor, nach der das Wasser der Hirnhöhlen kontinuierlich organisiert werde, ohne doch jemals organisiert zu sein. In einem späteren Briefe an Sömmering (4.August 1800) beteuert er, daß er nie daran gedacht habe, sein Werk als etwas «Absurdes» hinzustellen.

Goethe, dem Sömmering ebenfalls sein Buch zugesandt hatte, war in seiner Kritik viel deutlicher. Er bedauerte es lebhaft, daß Sömmering es nicht bei der Darstellung seiner anatomischen Befunde hatte bewenden lassen und die Philosophen herangezogen hatte. Er hätte seinem Buche den Titel «Von den Hirnenden der Nerven» geben sollen. Jeder hätte sich für die mannigfaltige Belehrung, die er der Darstellung der Natur entnommen, dankbar erwiesen. Dabei hätte er es vermieden, der Polemik anheimzufallen. Die Anatomen Camper und Blumenbach schwiegen sich aus. Der Publikumserfolg blieb aus.

Sömmering war kein philosophischer Kopf. Er gab sich bloß impulsiv dem Zeitgeiste hin, übte aber in seinem Denken zu wenig Selbstdisziplin, um den lockenden Autismen aus dem Wege zu gehen. Er war das Opfer seiner überschäumenden Begabung, die J. G. Zimmermann 1778 in einem Empfehlungsschreiben an CAMper so anschaulich geschildert hatte: SömMERING sei mit einer erstaunlichen Lebhaftigkeit im Erfassen aller Dinge begabt; er besitze einen ausgesprochenen Erfinder- und Entdeckergeist.

Sömmering hat eine Anzahl kleinerer Artikel verfaßt, von denen zwei die eben angedeuteten Entgleisungen aufweisen. Der erste betrifft die Frage, ob der durch die Guillotine vom Körper abgetrennte Kopf noch auf Sinnes- 
eindrücke reagieren könne. OELSNer veröffentlichte 1795 Sömmerings bejahende Antwort. Der zweite stammt aus Sömmerings Münchner Zeit. Man sandte ihm den auf dem Salzburger Friedhofe ausgegrabenen vermeintlichen Paracelsus-Schädel zur Begutachtung. Sömmering fand eine Fissur am Parietale, die er als intra vitam entstanden diagnostizierte. Er verlieh damit dem Märchen vom gewaltsamen Tode Hohenheims neue Nahrung. Bekanntlich zeigten die später ausgegrabenen wirklichen Überreste des Paracelsus keine Anzeichen einer Verletzung.

Als Sömmering nach vielem Ungemach schließlich in München als Anatom amtete, erwies er sich bald als einer der gefeiertsten Vertreter seines Faches in Deutschland. Bezeichnend dafür ist der Besuch des auf jede Belehrung erpichten napoleonischen Armeechirurgen Dominique LARReY beim «berühmten» Zergliederer Sömmering 1809. Aus diesem Zusammentreffen entwickelte sich eine aufrichtige Freundschaft zwischen den beiden, die so weit ging, daß sich LARrey beharrlich bei NApoleon und bei der Pariser Akademie für Sömmerings Entdeckung des Telegraphen verwendete. Sömmering hatte bekanntlich seit 1801 intensiv mit der Voutaschen Säule experimentiert und war auf eine Anregung des Ministers Montgelas hin auf den Einfall gekommen, den Telegraphen mittels Gasentbindung in Form eines Signalapparates zu verwirklichen. Seine Apparatur ist in allen Physikbüchern abgebildet.

Goethe hat trotz seiner scharfen Kritik an der verunglückten Abhandlung über das Organ der Seele nie aufgehört, Sömmering seine Hochschätzung kundzutun. Als er ihn in Frankfurt besuchte und sich von ihm seine Präparate und Abbildungen des menschlichen Auges, die ihn wegen ihrer Beziehung zu seiner Farbenlehre interessierten, zeigen ließ, kargte er nicht mit seiner Bewunderung.

Schließlich, als Söмmering zu seinem fünfzigjährigen Doktorjubiläum festlich geehrt wurde, finden wir Goeтнe unter den Gratulanten. Er sandte ihm ein elegantes Etui, das vier silberne Medaillen enthielt, mit der Aufschrift: «Seinem erprobten Freunde und Studiengenossen Sömuering an dessen Jubeltage 7.April 1828 widmet die Denkmale des weimarischen Jubeljahres 1825 in treuer Anhänglichkeit GoEthe.»

Sömmering und seine Zeitgenossen sind Vertreter jener Epoche der deutschen Geistesgeschichte, in der die Leistungen der Mediziner in einem Mißverhältnisse zu denjenigen der Dichter und Philosophen standen. Sie bieten aber dem Medizinhistoriker interessante Seiten, die darzustellen dem Verfasser reizvoll erschienen ist. 


\section{Literatur}

Originalwerke von S.Tн. Sömmering, insbesondere: Über das Organ der Seele (Königsberg 1796). - Rudolf W WGNer, S.Th.Sömmerings Leben und Verkehr mit seinen Zeitgenossen (Leipzig 1844; enthält den Briefwechsel mit Forster, Goethe usw.). - WiLhelm Stricker, S.Th.Sömmering (Neujahrsblatt, Frankfurt 1862). - Georg Forster, Briefwechsel mit S.Th.Sömmering. Herausgegeben von H. HetrNer (Braunschweig 1877). Heinrich Reintjes. Aus Georg Forsters Leben und Schriften. Eine Einführung (1940, Manuskript im Besitz der Basler Universitätsbibliothek). - Immanuel Kant, Sämtliche Werke. Herausgegeben von Karl Rosenkrantz und Friedrich Wilh. Schubert (Leipzig 1842). - W. Windelband, Präludien (6. Auflage, Tübingen 1919). - Јон. von Kries, Immanuel Kant und seine Bedeutung für die Naturforschung der Gegenwart (Berlin 1924). GoEthe, Briefe an S.Th.Sömmering; Campagne in Frankreich (1792); Belagerung von Mainz (1793). - Јон.P. Ecкеrmann, Gespräche mit Goethe (Leipzig 1836, Tl. I). - W. Schener, Geschichte der deutschen Literatur (Berlin 1889). - K. SpIno, Goethe und die Biologie (Schweiz. med. Wschr. 1932). - Sebastian Hodel, Goethes Metamorphosengedanke in Mißgeburtenlehre und Erkenntnistheorie (Inauguraldiss. Basel 1948). 